\title{
Editorial
}

\section{Chagas Disease in HIV-Infected Patients: It's Time to Consider the Diagnosis}

\author{
Melissa S. Nolan ${ }^{1 *}$ and Natasha S. Hochberg ${ }^{2,3}$ \\ ${ }^{1}$ Arnold School of Public Health, University of South Carolina, Columbia, South Carolina; ${ }^{2}$ School of Medicine, Boston University, Boston, \\ Massachusetts; ${ }^{3}$ Boston Medical Center, Boston, Massachusetts
}

Chagas disease, caused by the parasite Trypanosoma cruzi, is a leading cause of heart failure in Latin America, with a significantly worse prognosis than other causes of cardiomyopathy. ${ }^{1}$ Unfortunately, less than $1 \%$ of infected persons in the United States and endemic countries receive antiparasitic treatment. $^{2,3}$ The lack of standardized surveillance and prompt treatment leaves infected individuals vulnerable to life-long chronic infection, which in $20 \%$ to $30 \%$ of infected individuals can manifest as gastrointestinal tract organomegaly or cardiac system abnormalities. In those who develop cardiac disease, illness manifests as dilated cardiomyopathy, thromboembolism, or sudden cardiac death. ${ }^{4}$ Immunocompromised patients can develop severe disease during the acute phase or reactivation at any point during their lifetime. Reactivation typically manifests as myocarditis or central nervous system (CNS) disease, and should be considered in those diagnosed with HIV infection, or those undergoing cancer chemotherapy or receiving organ or bone marrow transplantation. ${ }^{5}$

This neglected tropical disease has increasingly become globalized as immigrant populations from endemic areas of Central and South America establish themselves in the United States, Europe, Japan, and elsewhere. ${ }^{6}$ More than 300,000 infected immigrants live in the United States, and more live in Spain and other non-endemic countries, ${ }^{7}$ highlighting the need for expansive awareness among the medical community. Mounting evidence supports Chagas disease surveillance in Latinx immigrants in the United States, where up to $20 \%$ of cases presenting with classic Chagas disease clinical signs and symptoms or epidemiological risk factors are misdiagnosed. ${ }^{8,9}$ As the recent coronavirus disease 2019 pandemic has taught us, these vulnerable groups are particularly susceptible to poor health-care access, and the imperative to diagnose this chronic, silent killer has never been greater.

HIV-infected patients are particularly susceptible to $T$. cruzi reactivation, manifesting as meningoencephalitis, CNS chagomas, acute myocarditis, and other sequelae. ${ }^{10}$ Although patients with reactivation disease can be treated with standard therapy (e.g., benznidazole), ${ }^{11}$ delayed diagnosis can prove fatal. An article in this issue of the American Journal of Tropical Medicine and Hygiene highlights the importance of heightened Chagas disease surveillance among HIVinfected patients. Reimer-McAtee et al. $^{12}$ conducted a cross-sectional study of $T$. cruzi prevalence among a Bolivian cohort of ambulatory and hospitalized HIV-infected individuals, identifying $28 \% T$. cruzi seropositivity, and reactivation disease in $12.5 \%$ of $T$. cruzi seropositive individuals. They present compelling evidence that, regardless of specific signs or

*Address correspondence to Melissa S. Nolan, Arnold School of Public Health, University of South Carolina, 915 Greene St., \#435D, Columbia, SC 29208. E-mail: msnolan@mailbox.sc.edu symptoms, HIV-infected patients from endemic countries should be tested for Chagas disease.

A challenge in treating HIV-infected patients with $T$. cruzi infection is understanding the parasite burden and whether CNS reactivation is imminent. Reimer-McAtee et al. used quantitative polymerase chain reaction ( $\mathrm{qPCR}$ ) to detect high levels of parasitemia, and demonstrated that these correlated with lower CD4 T-cell counts. These results suggest that qPCR may be used in conjunction with direct microscopy to suggest imminent reactivation disease. The current HIV treatment guidelines from the U.S. CDC, NIH, and the HIV Medicine Association of the Infectious Diseases Society of America suggest that qPCR assays can be performed on serial blood specimens in individuals infected with HIV and T. cruzi, and that an increasing parasite burden over time suggests reactivation. ${ }^{13}$ Furthermore, Reimer-McAtee et al. demonstrated that lower CD4 T-cell counts were associated with increased risk of reactivation disease. Notably, one of the outstanding questions in clinical care for HIV-infected individuals with Chagas disease is when to start combination antiretroviral therapy vis-à-vis anti-trypanosomal therapy. Current recommendations suggest no contraindication to concurrent treatment, but these patients should be monitored closely for possible immune reconstitution inflammatory syndrome. Furthermore, as a result of the elevated risk of treatment failure in HIVinfected patients, qPCR monitoring after treatment completion is recommended to offer an earlier indicator of treatment failure compared with serological conversion. ${ }^{14}$

Reimer-McAtee et al. highlight the burden of Chagas disease in Bolivians. Bolivia has a long history of active T. cruzi transmission, and its emigrant population has demonstrated high seropositivity years after migration. ${ }^{15}$ The lack of readily available treatment of Chagas disease in Bolivia ${ }^{3}$ renders HIV-infected individuals still living in Bolivia particularly vulnerable to $T$. cruzi reactivation. Furthermore, most Central and South American countries have high HIV-Chagas disease burdens, ${ }^{16}$ and the results of Reimer-McAtee et al. have important implications for these populations.

Despite their uniquely high seropositivity, Chagas disease surveillance in the United States should not be limited to Bolivian immigrants. Studies in the United States suggest overall seroprevalence rates of $1 \%$ to $3.8 \%,{ }^{17,18}$ higher rates in family members of those with Chagas disease (7.4\%), ${ }^{19}$ and up to $19 \%$ rates in Latinx immigrants with evidence of cardiomyopathy. ${ }^{8,9}$ Importantly, diagnosis and treatment of women in child-bearing years prevent congenital transmission ${ }^{20}$; lack of testing and treating infected women globally will result in new pediatric cases in endemic countries and among immigrants. Furthermore, a new wave of vector-acquired infections is potentially occurring in Latin America following the eradication of Rhodnius prolixus. ${ }^{21}$ Vector-borne disease transmission was believed to have been greatly interrupted as a result of the eradication of this key vector; however, new evidence 
suggests that transmission from non-eradicated secondary vectors (e.g., Triatoma dimidiata) is occurring and will result in new pediatric cases. ${ }^{16,22}$ Collectively, these two aforementioned factors highlight the need to consider children, not just adults, for Chagas disease diagnosis.

Reimer-McAtee et al. bring new attention to Chagas disease, an underdiagnosed problem, especially outside endemic regions. Clinicians in endemic countries and those working with Latinx patients more widely, particularly HIVinfected patients, should consider Chagas disease routinely in their differential diagnosis and clinical practice. Perhaps by raising awareness about Chagas disease among HIV providers, this neglected tropical disease can emerge from obscurity. Development of diagnostic and treatment guidelines by professional organizations such as the Infectious Diseases Society of America and the American Society of Tropical Medicine and Hygiene would help promote this goal.

Received June 7, 2021. Accepted for publication June 14, 2021.

Published online August 16, 2021.

Authors' addresses: Melissa S. Nolan, Arnold School of Public Health, University of South Carolina, Columbia, SC, E-mail: msnolan@ mailbox.sc.edu. Natasha S. Hochberg, School of Medicine, Boston University, Boston, MA, and Boston Medical Center, Boston, MA, E-mail:nhoch@bu.edu.

This is an open-access article distributed under the terms of the Creative Commons Attribution (CC-BY) License, which permits unrestricted use, distribution, and reproduction in any medium, provided the original author and source are credited.

\section{REFERENCES}

1. Bocchi EA, Arias A, Verdejo H, Diez M, Gómez E, Castro P, 2013. The reality of heart failure in Latin America. J Am Coll Cardiol 62: 949-958.

2. Herwaldt BL, Dougherty CP, Allen CK, Jolly JP, Brown MN, Yu P, Yu Y, 2018. Characteristics of patients for whom benznidazole was released through the CDC-sponsored investigational new drug program for treatment of Chagas disease-United States, 2011-2018. MMWR Morb Mortal Wkly Rep 67: 803-805.

3. Pinazo M-J et al., 2017. A strategy for scaling up access to comprehensive care in adults with Chagas disease in endemic countries: the Bolivian Chagas Platform. PLoS Negl Trop Dis 11: e0005770.

4. Buss LF, Bes TM, Pereira A, Natany L, Oliveira CDL, Ribeiro ALP, Sabino EC, 2021. Deriving a parsimonious cardiac endpoint for use in epidemiological studies of Chagas disease: results from the Retrovirus Epidemiology Donor Study-II (REDS-II) cohort. Rev Inst Med Trop São Paulo 63: e31.

5. Bern $C$ et al., 2007. Evaluation and treatment of Chagas disease in the United States: a systematic review. JAMA 298: 2171-2181.

6. Pino-Marín A, Medina-Rincón GJ, Gallo-Bernal S, Duran-Crane A, Arango Duque Ál, Rodríguez MJ, Medina-Mur R, Manrique FT, Forero JF, Medina HM, 2021. Chagas cardiomyopathy: from Romaña sign to heart failure and sudden cardiac death. Pathogens 10: 505.
7. Nunes MCP et al., 2018. Chagas cardiomyopathy: an update of current clinical knowledge and management: a scientific statement from the American Heart Association. Circulation 138: e169-e209.

8. Traina MI, Sanchez DR, Hernandez S, Bradfield JS, Labedi MR, Ngab TA, Steurer F, Montgomery SP, Meymandi SK, 2015. Prevalence and impact of Chagas disease among Latin American immigrants with nonischemic cardiomyopathy in Los Angeles, California. Circ Heart Fail 8: 938-943.

9. Nolan MS, Aguilar D, Misra A, Gunter SM, Erickson T, Gorchakov R, Rivera H, Montgomery SP, Murray KO, 2021. Trypanosoma cruzi in nonischemic cardiomyopathy patients, Houston, Texas, USA. Emerg Infect Dis 27: 1958-1960.

10. Bern C, Messenger LA, Whitman JD, Maguire JH, 2019. Chagas disease in the United States: a public health approach. Clin Microbiol Rev 33: e00023-19.

11. Yasukawa K, Patel SM, Flash CA, Stager CE, Goodman JC, WocColburn L, 2014. Trypanosoma cruzi meningoencephalitis in a patient with acquired immunodeficiency syndrome. Am J Trop Med Hyg 91: 84-85.

12. Reimer-McAtee MJ et al., 2021. HIV and Chagas disease: an evaluation of the use of real-time quantitative polymerase chain reaction to measure levels of Trypanosoma cruzi parasitemia in HIV patients in Cochabamba, Bolivia. Am J Trop Med Hyg 105: 643-650

13. Kaplan JE, Benson C, Holmes KK, Brooks JT, Pau A, Masur H, 2009. Guidelines for prevention and treatment of opportunistic infections in HIV-infected adults and adolescents: recommendations from CDC, the National Institutes of Health, and the HIV Medicine Association of the Infectious Diseases Society of America. MMWR Recomm Rep 58: 1-207, quiz, CE1-CE4.

14. Sulleiro $E$ et al., 2020. Usefulness of real-time PCR during followup of patients treated with benznidazole for chronic Chagas disease: experience in two referral centers in Barcelona. PLoS Negl Trop Dis 14: e0008067.

15. Conners EE, Vinetz JM, Weeks JR, Brouwer KC, 2016. A global systematic review of Chagas disease prevalence among migrants. Acta Trop 156: 68-78.

16. 2015. Chagas disease in Latin America: an epidemiological update based on 2010 estimates. Wkly Epidemiol Rec 90: 3343.

17. Meymandi SK, Forsyth CJ, Soverow J, Hernandez S, Sanchez D, Montgomery SP, Traina M, 2017. Prevalence of Chagas disease in the Latin American-born population of Los Angeles. Clin Infect Dis 64: 1182-1188.

18. Castro-Sesquen YE et al., 2021. Use of a latent class analysis in the diagnosis of chronic Chagas disease in the Washington metropolitan area. Clin Infect Dis 72: e303-e310.

19. Hernandez S, Forsyth CJ, Flores CA, Meymandi SK, 2019. Prevalence of Chagas disease among family members of previously diagnosed patients in Los Angeles, California. Clin Infect Dis 69: 1226-1228.

20. Stillwaggon E, Perez-Zetune V, Bialek SR, Montgomery SP, 2018. Congenital Chagas disease in the United States: cost savings through maternal screening. Am J Trop Med Hyg 98: 17331742.

21. Yoshioka K, Provedor E, Manne-Goehler J, 2018. The resilience of Triatoma dimidiata: an analysis of reinfestation in the Nicaraguan Chagas disease vector control program (2010-2016). PLoS One 13: e0202949.

22. Nolan MS et al., 2021. Elevated pediatric Chagas disease burden complicated by concomitant intestinal parasites and malnutrition in El Salvador. Trop Med Infect Dis 6: 72. 